\title{
Zinc finger protein 307 functions as a tumor-suppressor and inhibits cell proliferation by inducing apoptosis in hepatocellular carcinoma
}

\author{
YONGHAO LIANG* , QISHENG LI* , KELI CHEN, WEN NI, ZETAO ZHAN, FENG YE, YIYI LI, \\ YUAN FANG, FENGJIAO ZHANG, LONGHUA CHEN and YI DING \\ Department of Radiation Oncology, Nanfang Hospital, Southern Medical University, \\ Guangzhou, Guangdong 510515, P.R. China
}

Received January 8, 2017; Accepted July 4, 2017

DOI: $10.3892 /$ or.2017.5868

\begin{abstract}
Zinc finger protein 307 (ZNF307) is a new Krüppel-associated box zinc-finger protein gene and a member of the zinc-finger family of transcriptional factors. Notably, the role of ZNF307 and its underlying mechanisms involved in hepatocarcinogenesis are poorly investigated. In the present study, we found that the expression of ZNF307 was significantly downregulated in hepatocellular carcinoma (HCC) tissues, compared with that in adjacent non-tumor tissues. In vitro studies further demonstrated that ectopic expression of ZNF307 in HCC cell lines Bel7402 and HCCLM3 significantly reduced cell proliferation, migration and invasive ability. Concordantly, knockdown of ZNF307 increased cell proliferation, migration and invasive ability of HCC cell lines MHCC97L and QGY7701. In vivo functional studies showed that upregulation of ZNF307 expression in Bel7402 cells led to a suppression of tumorigenicity in mice, while knockdown of ZNF307 in MHCC97L cells resulted in reverse. effects. Importantly, flow cytometric analysis showed that ZNF307 overexpression increased the incidence of apoptosis, while ZNF307 knockdown decreased the incidence of apoptosis. Consistently, key regulators in apoptosis, such as caspase-3, BAX and BCL-2 were also regulated by ZNF307. Therefore, our results indicate that ZNF307 may serve as a tumor suppressor and inhibits cell proliferation of HCC via inducing apoptosis.
\end{abstract}

\section{Introduction}

Hepatocellular carcinoma (HCC) is the second leading cause of cancer-related death among males worldwide as well as in

Correspondence to: Dr Yi Ding or Dr Longhua Chen, Department of Radiation Oncology, Nanfang Hospital, Southern Medical University, Guangzhou, Guangdong 510515, P.R. China

E-mail: dingyi197980@126.com

E-mail: chenlhsmu@126.com

*Contributed equally

Key words: hepatocellular carcinoma, zinc finger protein 307 less developed countries. It is estimated that $\sim 782,500$ new liver cancer cases and 745,500 deaths occurred worldwide during 2012, with China alone accounting for $\sim 50 \%$ of the total number of cases and deaths (1). Despite numerous studies on multiple genes of HCC and advancements in the survival rate of HCC patients over the past decades (2), the exact molecular mechanisms underlying cancer initiation and progression are still unclear. Therefore, identification of novel HCC-associated molecules may provide insight into understanding the mechanism of hepatocarcinogenesis.

The zinc-finger family of proteins is one of the most common families of transcription factors in eukaryotic cells and has more than 3,000 members in the human genome $(3,4)$. Approximately one-third of zinc-finger proteins contain the Krüppel-associated box (KRAB-ZNFs) and $51 \%$ of KRAB-ZNFs are located on chromosome 19q13 (5). The functions of zinc-finger proteins are of great diversity, including DNA recognition, RNA packaging, transcriptional activation and regulation of apoptosis (6). Due to their multiple functions, several zinc-finger proteins have been identified as tumor-suppressors or oncogenes in the development of tumors. For example, zinc-finger protein ZBTB20 has been demonstrated to be a potential oncogene in non-small cell lung cancer (NSCLC) and HCC $(7,8)$. In addition, zinc-finger protein X-linked (ZFX) has been identified as an oncogene in colorectal cancer and is associated with poor prognosis (9). In contrast, ZNF382, ZNF569, ZNF331 and ZNF545 have been identified as tumor-suppressors (10-13). However, the role of most zinc-finger family members in tumor development remains ambiguous including HCC (14). Therefore, identification of novel zinc-finger proteins associated with tumor development may contribute to the understanding of the mechanisms of HCC.

Zinc finger protein 307 (ZNF307), also known as ZKSCAN4 (zinc-finger with KRAB and SCAN domains 4), ZSCAN36 and ZNF427, cloned from human embryonic heart cDNA, is a zinc-finger protein gene consisting of a Krüppel-associated box-A box, a SCAN and a zinc-finger domains with seven Cys2His2. Initial study demonstrated that in HEK-293 cells ZNF307 suppressed the transcriptional activity of p53 and p21 by upregulating mRNA levels 
of p53-binding protein MDM2 (MDM2) and E1A binding protein p300 (EP300) (15). However, Ecker et al reported that ZNF307 was co-precipitated and interacted with the glucocorticoid receptor in HEK293 cells (16). In addition, recent studies indicated that ZNF307 was one of two susceptibility loci for schizophrenia at $6 \mathrm{p} 21-\mathrm{p} 22.1$ by a genome-wide association study (17). Later, ZNF307 was also identified as one of nine risk SNPs distilled from genome-wide association studies for schizophrenia (18). However, the role of ZNF307 in tumor development and progression is largely unknown.

In the present study, we detected the expression level of ZNF307 in HCC and investigated its biological functions and mechanisms in human HCC cell lines.

\section{Materials and methods}

Tissue samples. Thirty-three paired HCC and adjacent non-tumor tissues $(>2 \mathrm{~cm}$ distance from the margin of resection) were randomly collected from patients who had not been pretreated with radiotherapy or chemotherapy prior to hepatectomy at Nanfang Hospital, Southern Medical University from April 2006 to Dec 2008 after obtaining informed consent. Specimens were frozen at $-80^{\circ} \mathrm{C}$ for RNA and protein isolation. The protocol of the present study was approved by the Southern Medical University Ethics Committee.

Cells and cell culture. L02, MHCC97L, HCCLM3, Huh7, QGY7701, QGY7703, Bel7402 and Bel7404 cell lines were obtained from the American Type Culture Collection (ATCC; Manassas, VA, USA) or the Cell Bank of the Chinese Academy of Sciences (Shanghai, China). All cells were cultured in high glucose Dulbecco's modified Eagle's medium (DMEM) (Gibco, Carlsbad, CA, USA) supplemented with 10\% fetal bovine serum (Biological Industries, Beit HaEmek, Israel) at $37^{\circ} \mathrm{C}$ in a humidified $5 \% \mathrm{CO}_{2}$ atmosphere.

RNA extraction and reverse transcription PCR. Total RNA from tissues or cells was extracted using TRIzol reagent (Takara, Dalian, China), according to the manufacturer's protocol. cDNA was synthesized from $1 \mu \mathrm{g}$ of total RNA using a reverse transcriptase cDNA synthesis kit (Takara). Real-time PCR amplification was performed on the ABI 7500 Real-Time PCR System (Applied Biosystems, Foster City, CA, USA), using a SYBR-Green PCR kit (Takara). In brief, the reaction system (total volume $20 \mu \mathrm{l}$ ) containing $500 \mathrm{ng}$ cDNA, the forward primer, 5'-TCTCCCTTGGTGGTGAAATACA-3' and the reverse primer, 5'-TTCCCTCAGGTGGCATATCTT-3', was used to amplify a 98-bp PCR product for human ZNF307 (NCBI; gene ID, 387032). The cycling parameters were $94^{\circ} \mathrm{C}$ for $35 \mathrm{sec}, 55^{\circ} \mathrm{C}$ for $40 \mathrm{sec}$ and $72^{\circ} \mathrm{C}$ for $34 \mathrm{sec}$ for 40 cycles. Real-time PCR was also carried out using the primers for GAPDH to normalize each of the extracts for ZNF307. The results are expressed as the ratio of copies of target genes to GAPDH. Each experiment was repeated independently at least three times. Relative expression levels of genes were calculated and expressed as $2^{-\Delta \Delta \mathrm{Ct}}$.

Western blotting. Cells and tissues were lysed on ice in RIPA buffer, sonicated and protein concentrations were calculated using a bicinchoninic acid (BCA) kit (both from
KeyGen Biotech Co., Ltd., Nanjing, China). A total of $50 \mathrm{mg}$ of protein lysates was separated by sodium dodecyl sulphate-polyacrylamide gel electrophoresis (SDS-PAGE), and then transferred onto a polyvinylidene difluoride (PVDF) membrane (Millipore, Bedford, MA, USA) and blocked in $5 \%$ non-fat dry milk in Tris-buffered saline, pH $7.5(100 \mathrm{mM}$ $\mathrm{NaCl}, 50 \mathrm{mM}$ Tris and $0.1 \%$ Tween-20). Membranes were incubated with anti-ZNF307 polyclonal antibody (LifeSpan BioSciences, Seattle, WA, USA) and anti-GAPDH antibody (Proteintech, Wuhan, China) at $4^{\circ} \mathrm{C}$ overnight, and then their respective secondary antibody. Signals were detected by enhanced chemiluminescence (Fdbio Science, Hangzhou, China).

Establishment of ZNF307-overexpressing cells. A lentivirus containing the ZNF307 cDNA and a lentivirus only-containing pCDF1-MCS2-EF1-copGFP mock vector were purchased from GenePharma (Shanghai, China). Bel7402 and HCCLM3 cells were seeded into 24 -well plates to $30-40 \%$ confluence and transfected with the viral supernatant containing exogenous ZNF307 cDNA and negative control lentivirus, respectively. After $24 \mathrm{~h}$ of incubation, the medium was replaced with fresh $10 \%$ fetal bovine serum (FBS). The expression of report gene copGFP was examined after 3-4 days of transfection. These two groups of cells were selected using $5 \mu \mathrm{g} / \mu \mathrm{l}$ puromycin (MP Biomedicals, Santa Ana, CA, USA) for two weeks. Then, overexpression of ZNF307 was confirmed by real-time PCR and western blotting.

Establishment of ZNF307-knockdown cells. ZNF307 short hairpin RNAs (shRNAs) (5'-GGTTTCACTCGAACTTCAT-3') and negative control shRNA (5'-TTCTCCGAACGTGTCA CGTTTC-3') were designed and synthesized by GenePharma. MHCC97L and QGY7701 cells were seeded into 24-well plates to $30-40 \%$ confluence, and transfected with the viral supernatant containing exogenous ZNF307 shRNA and negative control lentivirus, respectively. After $24 \mathrm{~h}$ of incubation, the medium was replaced with fresh 10\% FBS. The expression of report gene copGFP was examined after 3-4 days transfection. These two groups of cells were selected using $5 \mu \mathrm{g} / \mu \mathrm{l}$ puromycin (MP Biomedicals) for two weeks. Then, knockdown efficiency was evaluated after selection by real-time PCR and western blotting.

Colony-formation assay. Target HCC cells, as well as their control cells, were added to different wells of a 6-well culture plate at a final density of $1 \times 10^{2}$ cells. After culturing for 2 weeks at $37^{\circ} \mathrm{C}$, the cells were washed twice with phosphate-buffered saline (PBS), fixed with methanol and stained with Giemsa solution. Colonies with $>50$ cells/colony were counted. The experiment was repeated three times independently and results are shown as the means \pm SEM.

Cell proliferation assay. Target HCC cells, as well as their control cells, were re-plated in 96-well plates at a final density of $1 \times 10^{3}$ cells. Proliferation was measured using the Cell Counting Kit-8 (CCK-8; Dojindo, Shanghai, China) according to the manufacturer's instructions from day 1 to 7 . The experiment was repeated three times independently and the results are shown as the means \pm SEM. 

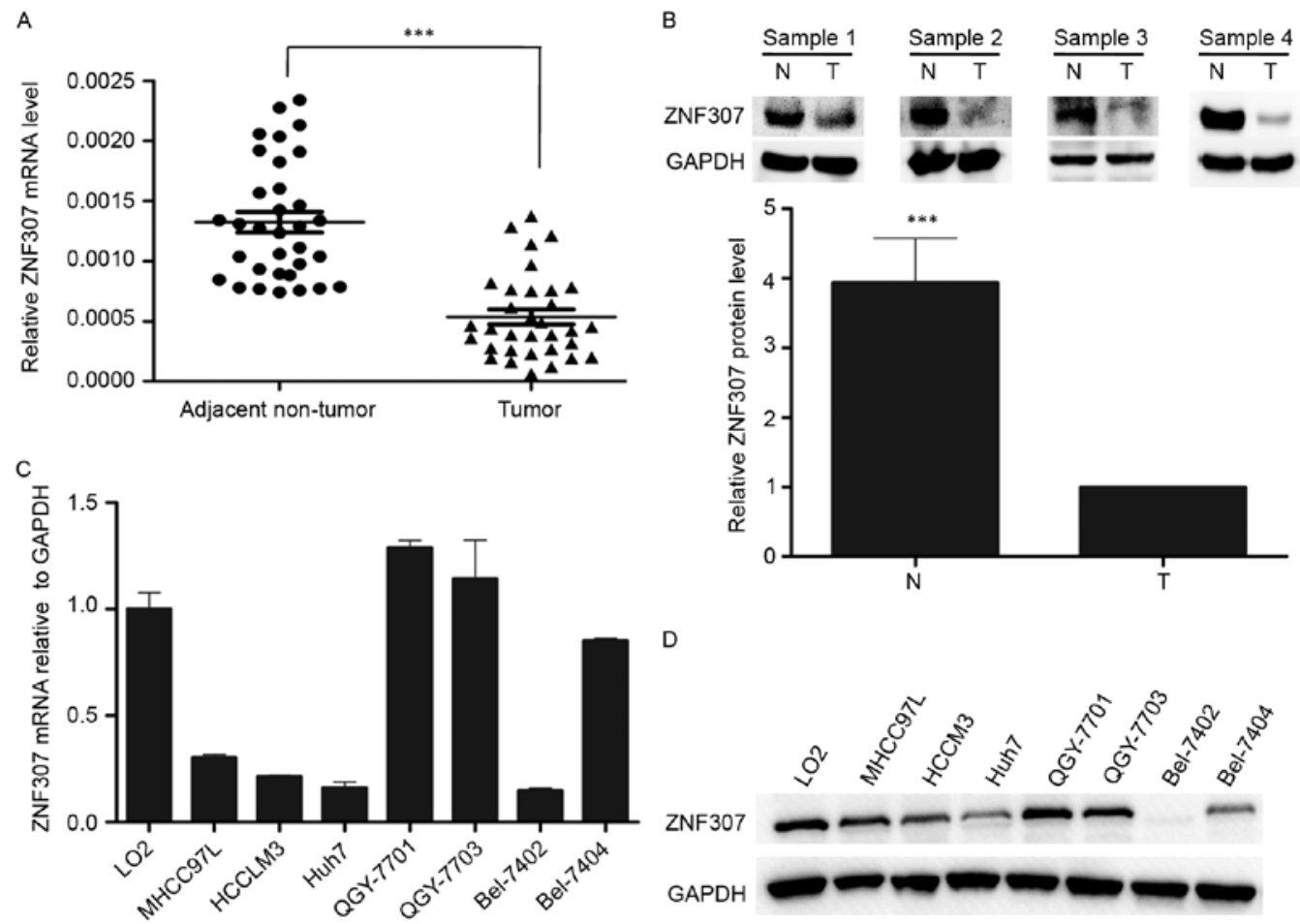

Figure 1. ZNF307 expression in HCC tissues and HCC cell lines. (A) Expression of ZNF307 mRNA in adjacent non-tumor tissues was significantly higher than that in tumor tissues, n=33. (B) Representative western blot analysis of ZNF307 expression in HCC and adjacent non-tumor tissues is shown and revealed that ZNF307 protein was expressed at a significantly higher level in adjacent non-tumor tissues (N) compared with tumor tissues (T); $\mathrm{n}=20$. (C and D) ZNF307 mRNA expression and protein expression in HCC cell lines and a normal liver cell line (L02). Data represent the mean \pm SEM of three independent experiments; ${ }^{* * *} \mathrm{P}<0.001$, in comparison with the control.

Wound healing and invasion assays. Cell migration ability was assessed using wound healing assays. When the cells grew to $80-90 \%$ confluence in 6 -well culture plates, three scratch wounds across each well were made using a $10-\mu 1$ pipette tip. The phase contrast images of the wounds were recorded at 0,24 and $48 \mathrm{~h}$, respectively. Cells transfected with the empty vector served as the controls. Invasion assay was performed using a Matrigel invasion chamber (Falcon, Corning Inc., Corning, NY, USA) according to the manufacturer's instructions. A total of $1 \times 10^{5}$ cells suspended in a serum-free medium were then added to the upper chamber, while 10\% FBS was added in the lower chamber as chemoattractant. After $24 \mathrm{~h}$ of incubation, noninvasive cells remaining in the upper chamber were removed by cotton swabs and the lower surface of membranes was stained with Giemsa solution. The permeating cells were counted under a inverted microscope and photographed in at least six random microscopic fields. The experiment was repeated three times independently and results are shown as the means \pm SEM.

In vivo tumorigenicity. Four- to six-week-old female BALB/c nude mice were purchased from the Central Laboratory of Animal Science at Southern Medical University (Guangzhou, China) and maintained in laminar-flow cabinets under specific pathogen-free conditions. A total of $1 \times 10^{7}$ targeted cells in $200 \mu \mathrm{l}$ PBS were subcutaneously injected into both flanks of nude mice. Tumor size was measured by calipers every 5 days, up to 25 days after injection. Tumor volume was calculated using the equation: $\mathrm{V}=\left[\right.$ length $\mathrm{x}$ (width) $\left.{ }^{2}\right] / 2$. The experiments were performed in seven mice in each treatment group.
Apoptosis analysis. Apoptosis assay was performed according to the manual of the Annexin V-FITC/propidium iodide (PI) apoptosis detection kit (KeyGen Biotech Co., Ltd.). Briefly, the harvested cells were washed twice with PBS, and then resuspended in binding buffer. Then, these cells were stained with Annexin V-FITC and PI at room temperature in the dark for $15 \mathrm{~min}$. In addition, these stained cells were analyzed using flow cytometry as soon as possible (within $1 \mathrm{~h}$ ). The experiment was repeated three times independently and results are shown as the means \pm SEM.

Statistical analysis. All measurements or variables are shown as means \pm SEM. Real-time PCR, western blotting, clone formation, wound healing, invasion and apoptosis assays were examined using the Student's t-test. To test statistical differences between in vitro cell proliferation and in vivo tumorigenicity, multiple-factor repetitive measurement and analysis of variance were performed. A difference was considered statistically significant at P-value $<0.05$. All statistical analyses were conducted using SPSS software (version 20; SPSS, Inc., Chicago, IL, USA).

\section{Results}

Downregulation of expression levels of ZNF307 mRNA and protein in HCC. Firstly, ZNF307 mRNA levels were measured in 33-paired HCC and adjacent non-tumor tissues by real-time PCR. The result revealed that ZNF307 mRNA levels were significantly downregulated in the HCC tissues $(\mathrm{P}<0.001$; Fig. 1A). In addition, western blot analysis of 20-paired 

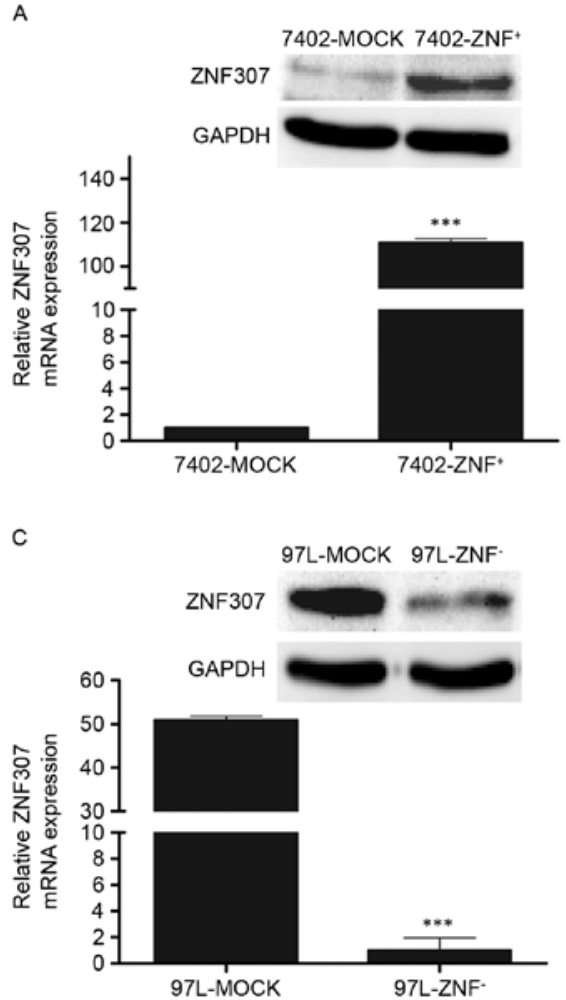
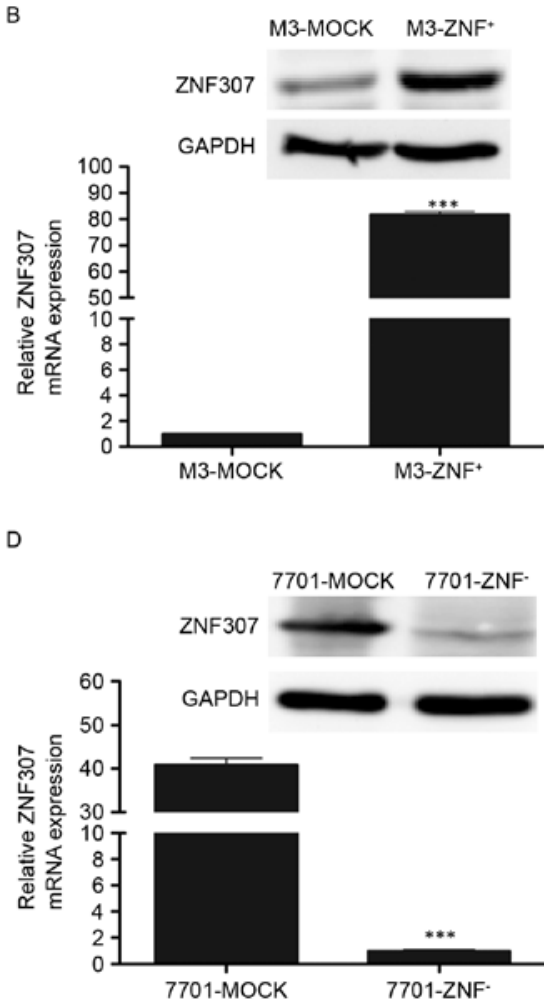

Figure 2. Overexpression and knockdown of ZNF307 in HCC cell lines. (A) RT-PCR and (B) western blot analyses confirmed the transfection and overexpression efficiencies of ZNF307 in Bel7402 (7402-ZNF+) and HCCM3 (M3-ZNF ${ }^{+}$cell lines. (C) RT-PCR and (D) western blot analyses revealed efficient knockdown of ZNF307 in MHCC97L (97L-ZNF) and QGY7701 (7701-ZNF) cell lines. Data represent the mean \pm SEM of three independent experiments; ${ }_{* * * *} \mathrm{P}<0.001$, in comparison with the control.

HCC tissues also confirmed the decreased protein abundance of ZNF307 in HCC tissues $(\mathrm{P}<0.001$; Fig. 1B). Moreover, we detected the ZNF307 mRNA expression in one human normal liver cell line (L02) and seven HCC cell lines (MHCC97L, HCCLM3, Huh7, QGY7701, QGY7703, Bel7402 and Bel7404). The results showed that ZNF307 was significantly downregulated in most of the HCC cell lines (Fig. 1C). The western blot analysis had a result similar to that of real-time PCR (Fig. 1D).

Alteration of ZNF307 expression regulates proliferation, migration, invasion and apoptosis of HCC cell lines in vitro. We successfully constructed two stable clones overexpressing ZNF307 from parental Bel7402 and HCCLM3 cells. Expression of ZNF307 after stable transfection was measured by RT-PCR and western blotting $(\mathrm{P}<0.001$; Fig. $2 \mathrm{~A}$ and $\mathrm{B})$. First, we found that expression of ZNF307 suppressed the growth of both Bel7402 and HCCLM3 cell lines by CCK-8 assay $(\mathrm{P}<0.001$; Fig. 3A). This was confirmed by colony formation assay. The colonies formed by Bel7402-ZNF307 ${ }^{+}$ $(\mathrm{P}<0.001)$ and HCCLM3-ZNF307 ${ }^{+}$cells $(\mathrm{P}<0.01)$ were significantly less and smaller than the control cells (Fig. 3B). In addition, ectopic expression of ZNF307 markedly slowed cell migration at the edges of the scratch wound in the Bel7402 and HCCLM3 cells. Quantitative analyses at $48 \mathrm{~h}$ confirmed a significant reduction in wound closure in the Bel7402-ZNF307 ${ }^{+}$ $(\mathrm{P}<0.001)$ and HCCLM3-ZNF307 ${ }^{+}$cells $(\mathrm{P}<0.01)$, compared with the controls cells (Fig. 3C). Furthermore, in the Matrigel invasion assay, the number of cells that passed through the Matrigel in the Bel7402-ZNF307 ${ }^{+}(\mathrm{P}<0.01)$ and
HCCLM3-ZNF307 ${ }^{+}$cells $(\mathrm{P}<0.001)$ was significantly less than in the mock cells (Fig. 3D). Moreover, Bel7402-ZNF307 ${ }^{+}$ and HCCLM3-ZNF307 ${ }^{+}$cells had an increased early and late apoptotic population compared to the control group $(\mathrm{P}<0.001$; Fig. 3E).

In contrast, the endogenous ZNF307 level in MHCC97L and QGY7701 cells was stably knocked down by transfection of shRNA $(\mathrm{P}<0.001$; Fig. $2 \mathrm{C}$ and $\mathrm{D})$. Contrary to the effect of ZNF307 overexpression, downregulation of endogenous ZNF307 in the MHCC97L and QGY7701 cells promoted the growth and colony formation compared with their controls $(\mathrm{P}<0.01$; Fig. $4 \mathrm{~A}$ and $\mathrm{B})$. Furthermore, an evident acceleration in the wound closure rate and enhanced invasiveness were observed in the MHCC97L-ZNF307and QGY7701-ZNF307- cells compared with their controls ( $\mathrm{P}<0.001$; Fig. 4C and D).

However, the proportion of early and late apoptotic cells was significantly decreased in the MHCC97L-ZNF307and QGY7701-ZNF307- cells compared to the control group $(\mathrm{P}<0.001$; Fig. 4E).

ZNF307 suppresses tumorigenicity in vivo. The effect of ZNF307 on in vivo tumorigenicity was investigated using a xenograft model in which nude mice were subcutaneously injected with Bel7402-ZNF+ ${ }^{+}$MHCC97L-ZNF ${ }^{-}$and their controls. We found that the volume of the tumors harvested from Bel7402-ZNF ${ }^{+}$cells was much smaller than that from the control by day 25 after injection $(\mathrm{P}<0.001$; Fig. 5A). In contrast, injection of MHCC97L-ZNF- cells led to obvious 

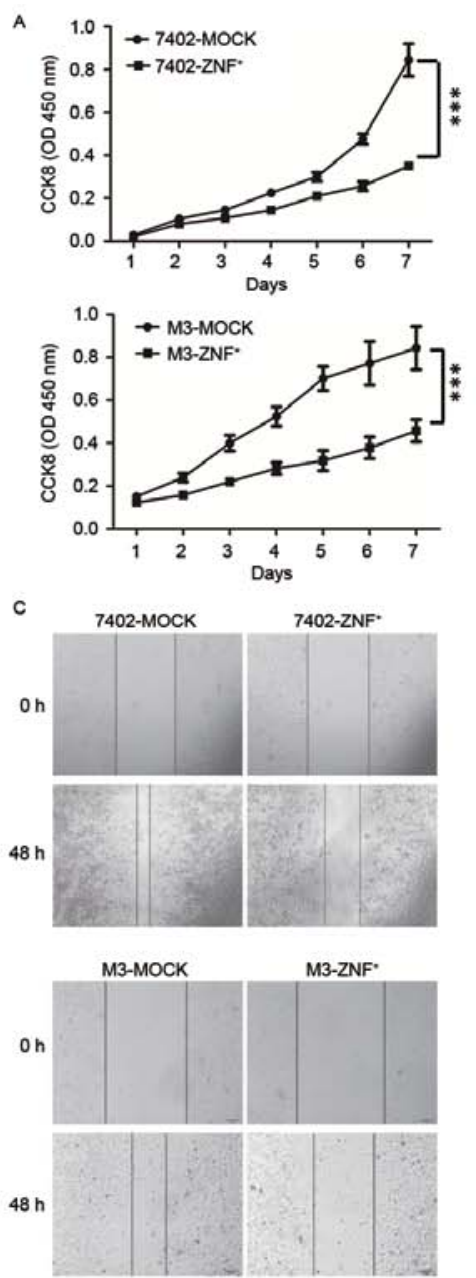
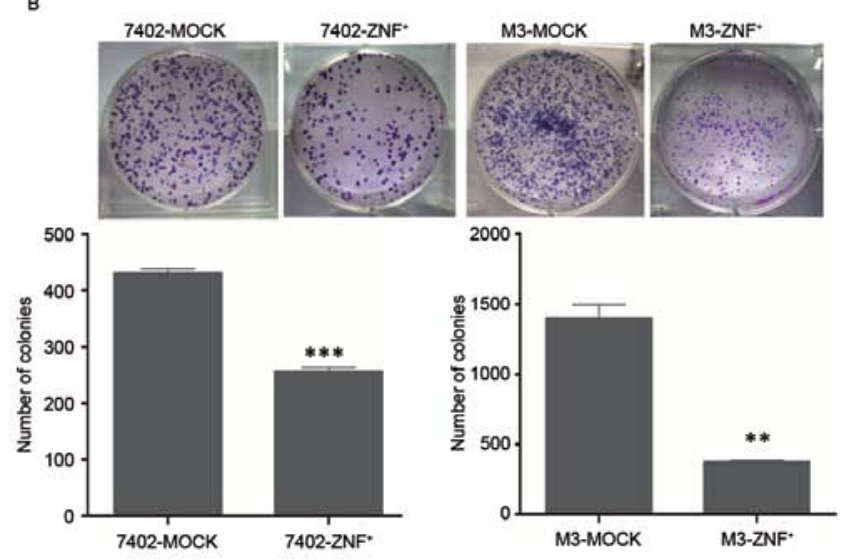

E
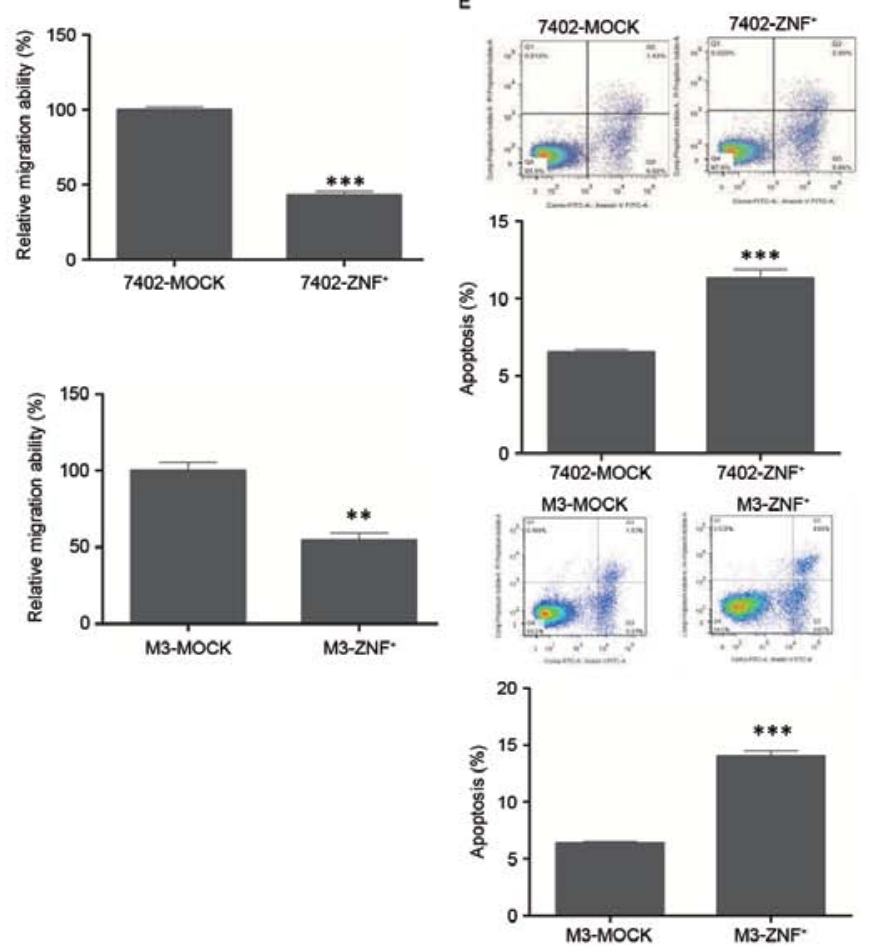
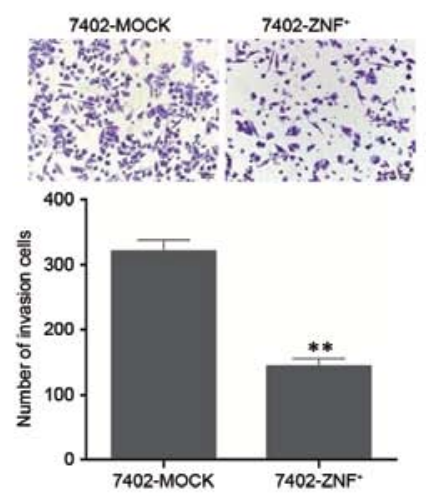

M3-MOCK

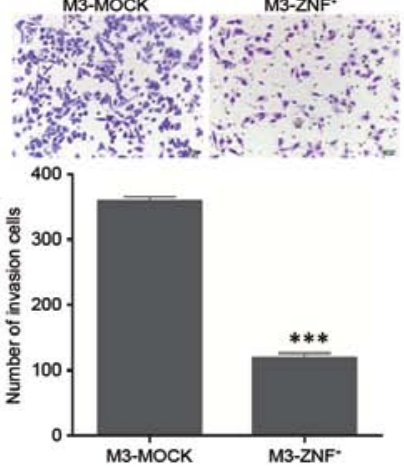

M3-ZNF 

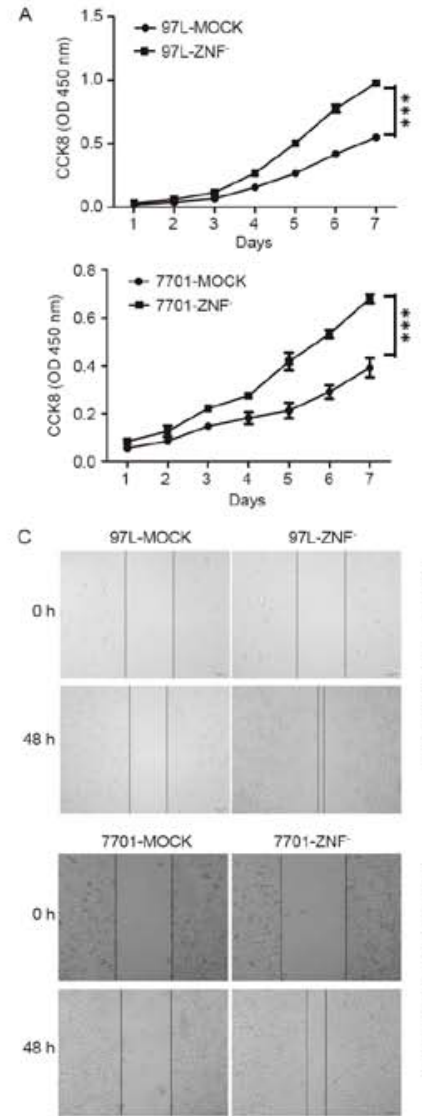
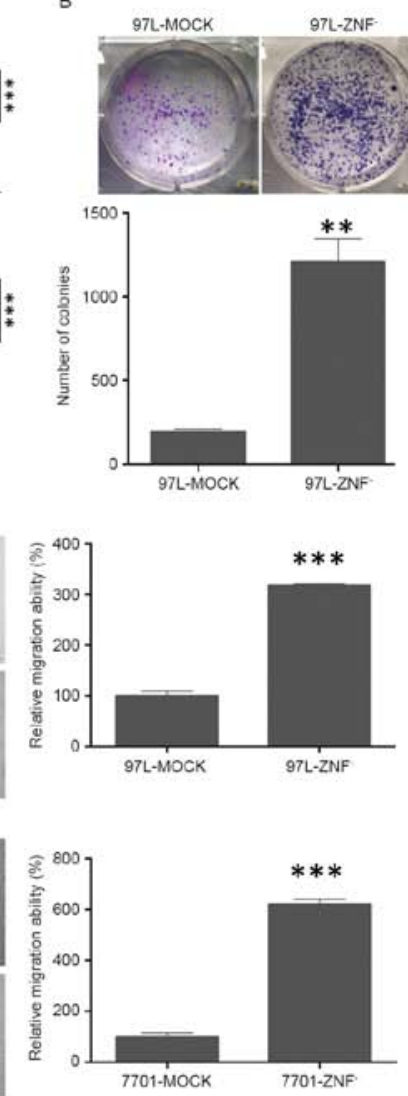
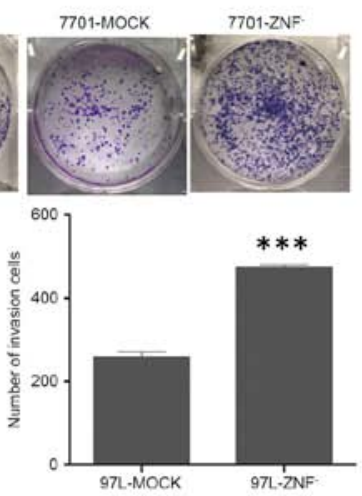

E
D
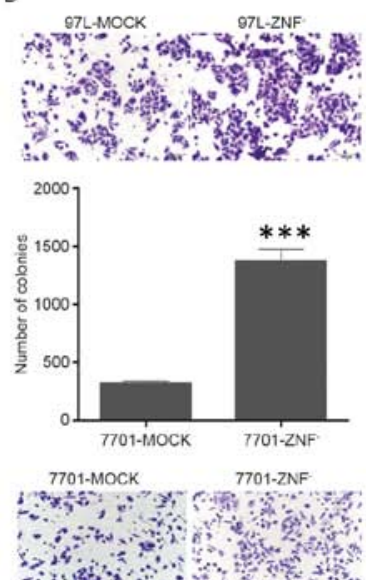

,

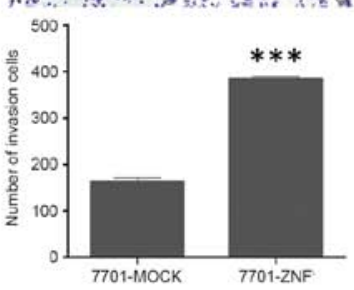
moted the cell proliferation of MHCC97L (97L-ZNF-) and QGY7701 (7701-ZNF-) cell lines as analyzed by CCK-8 assay. (B) ZNF307 knockdown enhanced the ability of colony formation in the $97 \mathrm{~L}-\mathrm{ZNF}^{-}$and $7701-\mathrm{ZNF}^{-}$cells, as analyzed by colony formation assay. (C) ZNF307 knockdown accelerated the ability of migration in the $97 \mathrm{~L}_{-} \mathrm{ZNF}$ and $7701-\mathrm{ZNF}^{-}$cells, as analyzed by wound-healing assay. (D) ZNF307 knockdown increased the number of invaded cells in the 97L-ZNF- and 7701-ZNF- cells, as analyzed by Matrigel invasion assay. (E) ZNF307 knockdown decreased the incidence of apoptosis in the 97L-ZNF ${ }^{-}$and $7701-\mathrm{ZNF}^{-}$cells, as analyzed using flow cytometric apoptosis assay. Data represent the mean \pm SEM of three independent experiments; ${ }^{* *} \mathrm{P}<0.01,{ }^{* * *} \mathrm{P}<0.001$, in comparison with the control (MOCK).

ZNF382, ZNF569, ZNF331 and ZNF545, have been identified as tumor-suppressors, whereas others, such as ZBTB20 and ZFX serve as oncogenic proteins (7-13).

ZNF307 is a novel zinc-finger protein and contains a Krüppel-associated box-A box, a SCAN and zinc-finger domains with seven Cys2His2. In HEK-293 cells, ZNF307 inhibits the transcriptional activity of p53 and p21 and interacts with the glucocorticoid receptor $(15,16)$. Recent two studies have demonstrated that ZNF307 is the susceptibility loci for schizophrenia by genome-wide association study $(17,18)$. Therefore, the role of ZNF307 in the development and progression of HCC is largely unknown.

In the present study, we examined the ZNF307 mRNA levels in 33-paired HCC and adjacent non-tumor tissues. The results revealed that ZNF307 mRNA levels were significantly downregulated in the HCC tissues. In addition, we confirmed that ZNF307 protein was expressed at a significantly lower level in the HCC tissues compared with that in the adjacent non-tumor tissues in 20-paired samples. Furthermore, we found that ZNF307 expression was significantly higher in the L02 cell line than that in seven HCC cell lines both at the mRNA and protein level. These results suggest that the downregulation of ZNF307 expression may contribute to HCC carcinogenesis.

In order to identify the function of ZNF307, we constructed four target cell lines: Bel7402-ZNF307 ${ }^{+}$, HCCLM3-ZNF307+, MHCC97L-ZNF- and QGY7701-ZNF- using a retroviral gene transfer method. We observed an association between the upregulation of ZNF307 and suppression of cell proliferation, migration and invasion in vitro, as well as an inverse association following knockdown of ZNF307. In addition, upregulation of ZNF307 expression in Bel7402 cells was related to suppression of tumorigenicity in mice. Concordantly, downregulation of ZNF307 expression in MHCC97L cells led to an enhancement of tumorigenicity in vivo. Importantly, flow cytometric analysis showed that ZNF307 overexpression increased the 
A
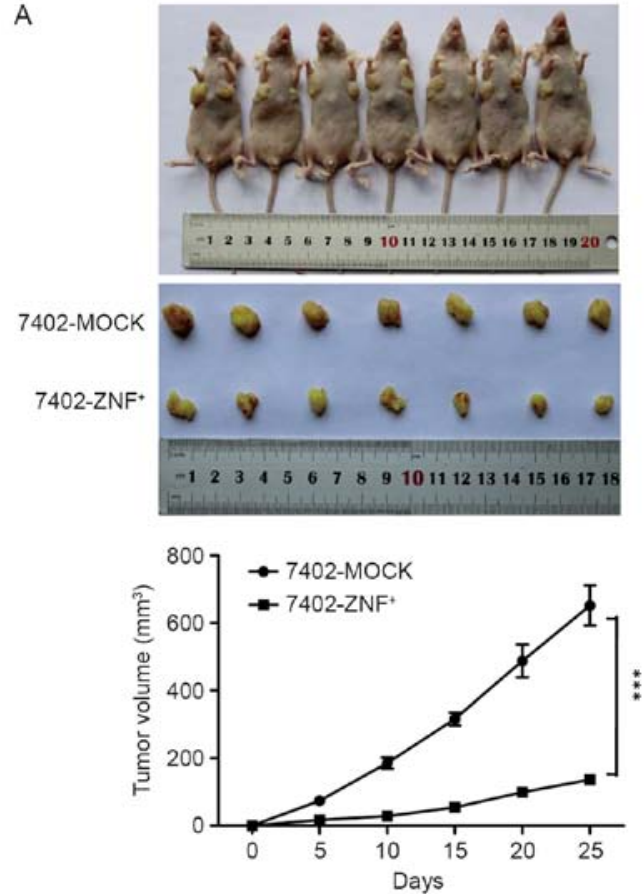

B
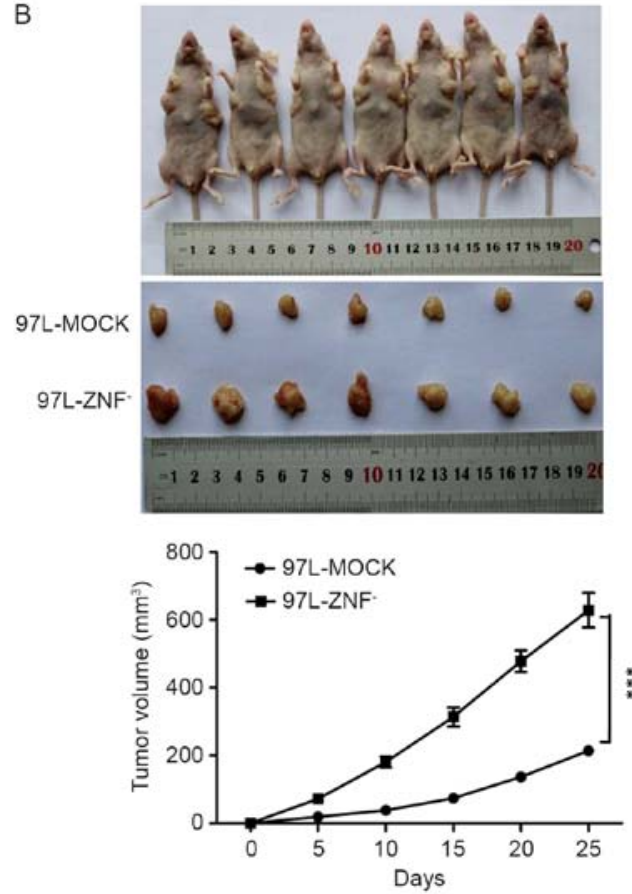

Figure 5. Effect of ZNF307 on subcutaneous tumorigenicity in HCC cell lines. (A) Overexpression of ZNF307 significantly inhibited the growth of Bel7402 $\left(7402-\mathrm{ZNF}^{+}\right.$) cells (mean tumor volume, $136.64 \mathrm{~mm}^{3}$ ) compared to the control group (mean tumor volume, $651.89 \mathrm{~mm}^{3}$ ). (B) Knockdown of ZNF307 significantly promoted growth of MHCC97L (97L-ZNF) cells (mean tumor volume, $628.46 \mathrm{~mm}^{3}$ ) compared to the control group (mean tumor volume, $214.35 \mathrm{~mm}^{3}$ ). Data represent the mean $\pm \mathrm{SEM} ;{ }^{* * * *} \mathrm{P}<0.001$ by t-test, in comparison with control.

A

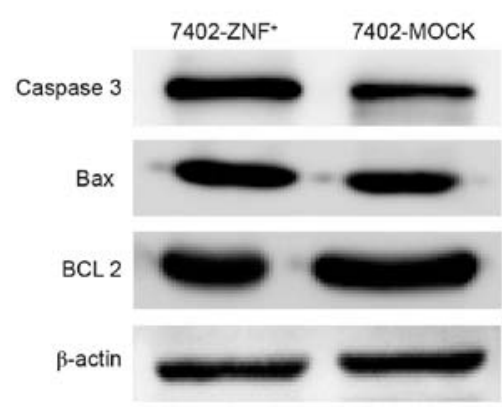

B

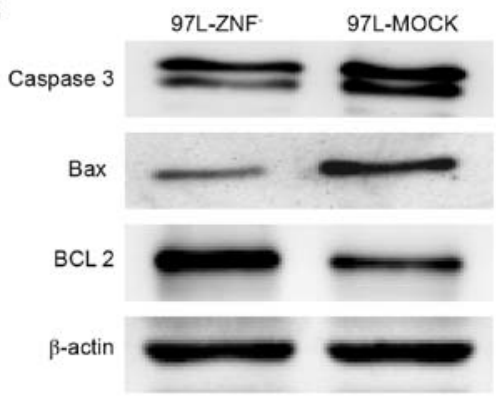

Figure 6. ZNF307 increases the expression of caspase-3 and BAX and decreases the expression of BCL-2. (A) Overexpression of ZNF307 increased caspase-3 and BAX, and decreased BCL-2 in the 7402-ZNF ${ }^{+}$cells, compared with the controls (MOCK). (B) Knockdown of ZNF307 upregulated Bcl-2, and downregulated caspase-3 and Bax in the 97L-ZNF- cells, compared with the controls (MOCK).

incidence of apoptosis, while ZNF307 knockdown decreased the incidence of apoptosis.

Given the flow cytometric apoptosis results, we examined the expression of apoptosis-related proteins in Bel7402-ZNF ${ }^{+}$ and MHCC97L-ZNF ${ }^{-}$cells by western blotting. We found that ZNF307 increased the transcription of Bax and caspase-3, and decreased the transcription of Bcl-2. Therefore, we confirmed that the suppression of HCC cell growth by ZNF307 was mediated by induction of apoptosis and by regulation of apoptosis-related proteins.

Unfortunately, we failed to examine the ZNF307 expression level of HCC tissues by immunohistochemistry. It is likely that the ZNF307 protein expression level was too low to be tested.

As a zinc-finger protein, ZNF307 may serve as a transcriptional repressor in HCC. However, due to the absence of specific chip-grade antibodies against ZNF307, the DNA sequences binding ZNF307 under a biological state could not be analyzed by ChIP or ChIP-seq. Systematic approaches (gene expression array or ChIP-seq) may be useful to study the function of ZNF307 and may reveal additional targets in the future.

According to the above mentioned results, ZNF307 may be a tumor-suppressor in HCC. However, Li et al found that in HEK-293 cells, ZNF307 suppressed the transcriptional activity of p53 and p21, which indicates that ZNF307 may enhance tumorigenesis. It is likely that ZNF307 may act as a tumor-suppressor or an oncogene in different systems. Several genes have been reported to function as a a tumorsuppressor or oncogene in different tumors. For example, miR-506 has been demonstrated to play a role as an oncogene in tumorigenesis and a tumor-suppressor in the progression 
in pancreatic ductal adenocarcinoma (20). Lo et al found that FOXF2 has a complex role in breast cancer and functions as either a tumor-suppressor or an oncogene depending on the breast tumor subtype (21). E2F1, a critical tumor-suppressor molecule in cell cycle progression and induction of apoptosis, has been identified to enhance invasion and metastasis by activating growth receptor signaling pathways (22). Therefore, all these attempts addressing the function of ZNF307 in carcinomas require further in-depth research in the future.

In the present study, we, for the first time, demonstrated that ZNF307 functions as a tumor-suppressor in HCC by inhibiting cell proliferation, migration and invasion, and it induced apoptosis by regulating various genes (caspase-3, Bax and Bcl-2). ZNF307 may serve as a potential tumor marker for HCC.

\section{Acknowledgements}

Grant sponsors of the present study were the Pearl River S\&T Nova Program of Guangzhou (grant no. 2014J2200015), Guangdong Natural Science Funds for Distinguished Young Scholars (grant no. 2015A030306015), the Excellent Young Teachers Program of Higher Education of Guangdong Province (grant no. YQ2015036), the Guangzhou Program for Support of Top-Notch Young Professionals (grant no. 2015TQ01R279) and the National Natural Science Foundation of China (grant no. 81672992).

\section{References}

1. Torre LA, Bray F, Siegel RL, Ferlay J, Lortet-Tieulent J and Jemal A: Global cancer statistics, 2012. CA Cancer J Clin 65: 87-108, 2015.

2. Kanda M, Sugimoto $\mathrm{H}$ and Kodera Y: Genetic and epigenetic aspects of initiation and progression of hepatocellular carcinoma World J Gastroenterol 21: 10584-10597, 2015.

3. Papavassiliou AG: Transcription factors: Structure, function, and implication in malignant growth. Anticancer Res 15: 891-894, 1995.

4. Ladomery M and Dellaire G: Multifunctional zinc finger proteins in development and disease. Ann Hum Genet 66: 331-342, 2002.

5. Urrutia R: KRAB-containing zinc-finger repressor proteins. Genome Biol 4: 231, 2003.

6. Laity JH, Lee BM and Wright PE: Zinc finger proteins: New insights into structural and functional diversity. Curr Opin Struct Biol 11: 39-46, 2001.

7. Zhao JG, Ren KM and Tang J: Zinc finger protein ZBTB20 promotes cell proliferation in non-small cell lung cancer through repression of FoxO1. FEBS Lett 588: 4536-4542, 2014.
8. Wang Q, Tan YX, Ren YB, Dong LW, Xie ZF, Tang L, Cao D, Zhang WP, Hu HP and Wang HY: Zinc finger protein ZBTB20 expression is increased in hepatocellular carcinoma and associated with poor prognosis. BMC Cancer 11: 271, 2011.

9. Jiang J and Liu LY: Zinc finger protein X-linked is overexpressed in colorectal cancer and is associated with poor prognosis. Oncol Lett 10: 810-814, 2015.

10. Cheng Y, Geng H, Cheng SH, Liang P, Bai Y, Li J, Srivastava G, $\mathrm{Ng} \mathrm{MH}$, Fukagawa T, Wu X, et al: KRAB zinc finger protein ZNF382 is a proapoptotic tumor suppressor that represses multiple oncogenes and is commonly silenced in multiple carcinomas. Cancer Res 70: 6516-6526, 2010.

11. Huang X, Yuan W, Huang W, Bai Y, Deng Y, Zhu C, Liang P, Li Y, Du X, Liu M, et al: ZNF569, a novel KRAB-containing zinc finger protein, suppresses MAPK signaling pathway. Biochem Biophys Res Commun 346: 621-628, 2006.

12. Yu J, Liang QY, Wang J, Cheng Y, Wang S, Poon TC, Go MY, Tao Q, Chang Z and Sung JJ: Zinc-finger protein 331, a novel putative tumor suppressor, suppresses growth and invasiveness of gastric cancer. Oncogene 32: 307-317, 2013.

13. Xiao Y, Xiang T, Luo X, Li C, Li Q, Peng W, Li L, Li S, Wang Z, Tang L, et al: Zinc-finger protein 545 inhibits cell proliferation as a tumor suppressor through inducing apoptosis and is disrupted by promoter methylation in breast cancer. PLoS One 9: e110990, 2014.

14. Jen $\mathrm{J}$ and Wang YC: Zinc finger proteins in cancer progression. $\mathrm{J}$ Biomed Sci 23: 53, 2016.

15. Li J, Wang Y, Fan X, Mo X, Wang Z, Li Y, Yin Z, Deng Y, Luo N, Zhu C, et al: ZNF307, a novel zinc finger gene suppresses p53 and p21 pathway. Biochem Biophys Res Commun 363: 895-900, 2007.

16. Ecker K, Lorenz A, Wolf F, Ploner C, Böck G, Duncan T, Geley $S$ and Helmberg A: A RAS recruitment screen identifies ZKSCAN4 as a glucocorticoid receptor-interacting protein. J Mol Endocrinol 42: 105-117, 2009.

17. Yue WH, Wang HF, Sun LD, Tang FL, Liu ZH, Zhang HX, Li WQ, Zhang YL, Zhang Y, Ma CC, et al: Genome-wide association study identifies a susceptibility locus for schizophrenia in Han Chinese at 11p11.2. Nat Genet 43: 1228-1231, 2011.

18. Ma L, Tang J, Wang D, Zhang W, Liu W, Wang D, Liu XH, Gong W, Yao YG and Chen X: Evaluating risk loci for schizophrenia distilled from genome-wide association studies in Han Chinese from Central China. Mol Psychiatry 18: 638-639, 2013.

19. Zhu RX, Seto WK, Lai CL and Yuen MF: Epidemiology of hepatocellular carcinoma in the Asia-Pacific region. Gut Liver 10: 332-339, 2016.

20. Cheng RF, Wang J, Zhang JY, Sun L, Zhao YR, Qiu ZQ, Sun BC and Sun Y: MicroRNA-506 is up-regulated in the development of pancreatic ductal adenocarcinoma and is associated with attenuated disease progression. Chin J Cancer 35: 64, 2016.

21. Lo PK, Lee JS, Liang X and Sukumar S: The dual role of FOXF2 in regulation of DNA replication and the epithelial-mesenchymal transition in breast cancer progression. Cell Signal 28: 1502-1519, 2016.

22. Engelmann D and Pützer BM: The dark side of E2F1: In transit beyond apoptosis. Cancer Res 72: 571-575, 2012. 